\title{
L os servicios bibliotecarios y de información en el contexto de la biblioteca tradicional 1
}

\author{
Georgina Araceli Torres Vargas \\ Centro Universitario de Investigaciones Bibliotecológicas \\ de la UNAM, 04510, México D .F., Tel: 56-23-03-29 \\ E-mail: arageo@yahoo.com
}

\begin{abstract}
RESUMEN
A través de un análisis histórico se aborda cómo se han gestado los servicios bibliotecarios y de información tradicionales, a qué objetivos han respondido, y cuáles son las características que éstos le han dado a la biblioteca tradicional. El análisis se muestra desde una perspectiva general, por lo que no se enuncia cada tipo de servicio. El objetivo primordial es caracterizar los servicios de la biblioteca tradicional.

Palabras Clave: Servicios de Información, Bibliotecas.
\end{abstract}

\section{LIBRARY AND INFORMATION SERVICES IN THE CONTEXT OF THE TRADICIONAL LIBRARY Georgina Araceli Torres-VARgaS}

\begin{abstract}
:
The paper examines through historical analysis the way in which the traditional library and information services were created, what purposes have they fulfilled and which are the characteristics that they have given to the traditional library. The analysis shows only a general perspective and does not include all types of services. Its main aim is to describe the services of the traditional library.

Key Words: Information Services, Libraries.
\end{abstract}

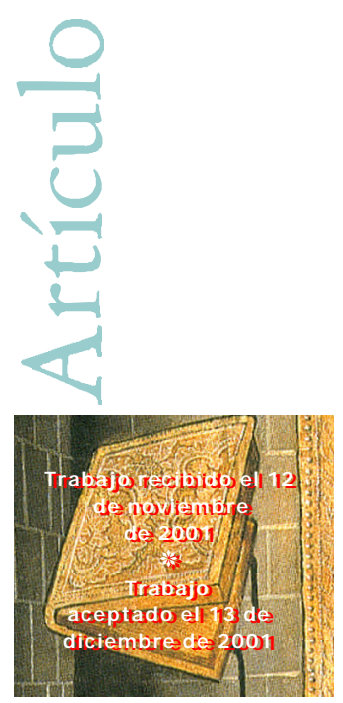

\section{INTRODUCCIÓN}

\begin{abstract}
on frecuencia se argumenta que uno de los fines de la biblioteca es el ofrecimiento de servicios, en tanto que sin ellos la información recopilada y organizada no tendría razón de ser y puesto que representan el puente de enlace entre la información y quien la necesita.
\end{abstract}

1 El presente artículo deriva de un proyecto de investigación apoyado por el Consejo Nacional de Ciencia y Tecnología. 
Sin embargo aun cuando se reconoce su importancia, en bibliotecología y ciencias de la información son escasos los estudios en que se abordan los servicios bibliotecarios desde su aspecto teórico. Q uizá esto se debaal enfoque preponderantemente práctico que se le da a este tema, ${ }^{2}$ lo que por ejemplo ha dado lugar a la elaboración de numerosos estudios de caso.

Sin embargo los estudios teóricos sobre los servicios parecen hoy más apremiantes dada la urgencia por comprender qué sucede en el medio digital.

Para indagar lo referente a los servicios de la biblioteca digital, se requiere analizar qué pasa con los servicios en el mundo de los impresos desde un punto de vista general. Un estudio de esta naturaleza implica conocer las circunstancias en las se han gestado los servicios que hoy conocemos, los o bjetivos a los que éstos responden y, sobre todo, las características que estos servicios le han asignado ala biblioteca tradicional.

Éstos son precisamente algunos de los fines del presente trabajo y por ello no le damos atención a cada uno de los servicios (llámense de consulta, de préstamo, etcétera); más bien, con base en una revisión histórica, se intenta observar la razón de ser de los servicios tradicionales en su conjunto.

Se espera que este acercamiento pueda servir como base para la reflexionar sobre los servicios de la biblioteca digital, objeto de estudio en mi proyecto de investigación en curso.

\section{SERVICIOSBIBLIOTECARIOSY SERVICIOSDE INFORMACIÓN}

En los artículos dedicados al estudio de los servicios no queda explícito a qué se refieren éstos cuando se habla de seviciobiblicteranioo de servidodeinformadón Sin embargo algunos autores ofrecen dar ciertas pautas quenos clarifican a esterespecto.

Buckland ${ }^{3}$ se refiere a los servicosbibidtearioscomo aquellos medios que permiten el acceso a la información que posee la biblioteca. Este acceso apoya la misión de la institución y los intereses de la población a la que sirve.

Con respecto al acceso, Mitchell 4 señala que es mediante éste como se permite el aprovechamiento o uso de las colecciones.

Considerados así, los servicios bibliotecarios serían el puente o canal que permite el acceso a los documentos de una biblioteca.

El accopouede ser visto desde dos perspectivas:

2 Ya que por lo general se describen los servicios de alguna biblioteca en particular y sus resultados de evaluación.

3 Buckland, Michael. ReekiginingLibrary Services A Manifesto

Disponible en: http: / / sunsite.berkeley.edu/ Literature/ Library/ Redesigning/ html.html

4 Mitchell W., Bede.A ccess: thekegtopubicsevice p. 1- 22. En Accessservicesinlibranies: nevsdutionsfor colletionmanaggnet// G regg Sall, editor. N.Y : Haworth, 1992. p. 1. 


\section{Investigacón Biblidteedógica v. 15 N o. 31 julio/ diciembre de 2001}

1. Como el proceso de aproximación a la información documental, es decir, la identificación de documentos a través de catálogos, repertorios bibliográficos, bases de datos, etcétera.

2. El acceso también puede implicar la disponibilidad 5 y el uso de la información. Hay disponibilidad cuando se tiene el documento físicamente al alcance, listo para su uso. La disponibilidad se relaciona estrechamente con el concepto de préstamo.

En los servicios bibliotecarios el acceso se da a partir de la identificación de los documentos de su colección (a través de medios como los catálogos y bibliografías, tanto impresos como en línea o en disco compacto) e incluye su posterior disponibilidad en la misma biblioteca.

Por su parte, de acuerdo con Foskett, los sevidiosdeinfamadónsurgen a partir de la necesidad de enterar a los investigadores sobre las nuevas publicaciones que se generan en su ámbito de acción, ${ }^{6}$ sin que éstas necesariamente formen parte de la colección de la biblioteca que las da a conocer. Para el autor los servicios de información se relacionan con la aparición de los catálogos de unión, ya que por medio de ellos se pueden dar a conocer los materiales que poseen otras bibliotecas.

Como puede verse los servicios bibliotecarios y los servicios de información se diferencian en razón de la disponibilidad de los materiales. Mientras los primeros permiten el uso de los materiales que posee físicamente la biblioteca, los segundos facilitan la identificación pero no necesariamente la disponibilidad inmediata de los documentos, puesto que éstos pueden estar en otras bibliotecas.

En el caso de la biblioteca tradicional podemos hablar tanto de servicios bibliotecarios como de servicios de información.

Si bien la aparición de las bases de datos en línea y en discos compactos dieron origen a lo que en un tiempo se llamó biblictecaautomatizada ésta puede ubicarse también en el contexto de la biblioteca tradicional. Ello obedece a que en la biblioteca automatizada se puede hacer uso de tecnologías como las bases de datos para identificar documentos, pero en su colección no se encuentran publicaciones electrónicas. Este sería el caso de la biblioteca electrónica.

\section{LA BIBLIOTECA TRADICIONAL VISTA A TRAVÉS DE ALGUNOS MODELOS}

Si se tipifica ala biblioteca con base en el grado tecnológico que muestre, desde la naturaleza de la colección hasta las tecnologías que se emplean para el manejo y uso de la información, podríamos hablar de la biblioteca tradicional y de la biblioteca del futuro.

5 Hay disponibilidad cuando se tiene el documento físicamente al alcance, por ejemplo, a través del servicio de préstamo.

6 Foskett, D.J. Information servicein libranies India : Akashdeep, 1992, p. 11. 
Peter Brophy7 señala que la biblioteca tradicional no corresponde a una sola imagen sino a muchasy que éstas están determinadas por los propósitos que las guían.

D e acuerdo con esto a partir de cada modelo de biblioteca tradicional pueden identificarse diferentes formas de servicios. Veamos primero los diferentes modelos de la biblioteca tradicional.

\section{La biblioteca bajo el modelo de acumulación}

El modelo de biblioteca más antiguo y perdurable, es aquel en el cual se la concibe como una colección de libros.

Los antecedentes más remotos de este modelo de biblioteca, se observan en la biblioteca de Ebla, ${ }^{8}$ la más antigua de la que se tiene noticia. Se piensa que dicha biblioteca apareció como respuesta a la necesidad de conservar la memoria escrita, y de contar con un sitio en donde albergar los documentos que registrasen lo concerniente a una cultura.

Aunque es poco lo que se conoce de la Biblioteca de Ebla podemos apreciar su importancia para el desarrollo de la biblioteconomía, y en cuanto a la idea de biblioteca resalta ésta como espacio para la conservación de los escritos.

Para muchos autores, entre ellos Brophy, una de las bibliotecas más representativas dentro de este modelo de acumulación es la biblioteca de Alejandría. Su colección de papiros y rollos llegó a cientos de miles de ejemplares, sin duda la más grande de la antigüedad.

Pero esta biblioteca estaba estrechamente vinculada a un instituto de investigaciones planeado por Ptolomeo I, por lo que su vasta colección no fue establecida sólo para reunir toda la literatura griega en las mejores copias disponibles, sino para apoyar a los investigadores en sus estudios. No sólo fue esta biblioteca el centro del helenismo, sino también de los estudios semíticos.

La biblioteca también se utilizaba como escuela y centro de investigaciones; era un santuario y una fuente de inspiración para eruditos. En ella floreció la cultura greco-romana y fue considerada como una de las fuentes más ricas de la cultura antigua, especialmente en lo relativo a ciencia y filosofía.

Ptolomeo I (Rey de Egipto 305-282 a. C.) y sus sucesores, establecieron en Alejandría una institución que puede ser vista como una biblioteca en el moderno sentido de la palabra, pues contaba con personal bajo las ordenes de un bibliotecario que adquiría y organizaba el material bibliográfico para el uso de lectores calificados. ${ }^{9} \mathrm{Es}$ por eso que la biblioteca de Alejandría no debeverse como un ejemplo de biblioteca cuyo propósito es la acumulación.

7 Crr. Brophy, Peter. Thelibraryin thetwenty first certury: newservicesfor theinfomationage London : Library Association Publishing, 2001.

8 Ebla fue una ciudad situada a unos ochenta kilómetros al sur de Alepo, cerca de Ugarit.

9 Arashanipalai Neelameghan. Coxpeacaónyasistemiaintemaaionales En:Infomemmolal sdrelainfomadón 1997-1998/ YvesCourrier y Andrew Large, ed. Madrid : UNESCO : CIND OC, 1997. p. 388. 
Alejandría no fue la única gran biblioteca del mundo helénico, existe también información acerca de la biblioteca de Pérgamo ${ }^{10}$ Esta biblioteca, considerada junto con la de Alejandría como una de las más grandes de la antigüedad, fue fundada por Eumenes II (197-159 a.C) en el templo de Minerva y su primer director fue Crates de Malos, un filósofo que alcanzó gran prestigio en Roma.

Los amplios cuartos de la biblioteca de Pérgamo contenían cerca de 80,000 rollos, visibles para el público, pero accesibles sólo para el personal.

Las bibliotecas de la Edad Media son ejemplos de bibliotecas cuyo propósito principal era la acumulación de obras.

Quizá ante el peligro que representaba para esa época y su cultura el avance del cristianismo, surgió entre los romanos un interés desmedido por perpetuar su literatura. Y para lograrlo recurrieron a la compilación de extractos y antologías, además de traducir lo más importante de la literatura y el pensamiento griego.

Una de las actividades más importantes para poder reunir los títulos fue sin duda la copia y producción de libros en el escritorio monacal, actividad a través de la cual se produjeron y copiaron numerosas obras de gran valor. ${ }^{11}$

\section{L a biblioteca basada en el registro de la información}

Lamayoría de los estudiosos de la historia de la bibliografía consideran que las bibliografías comenzaron con el surgimiento de la imprenta y que la primera bibliografía fue el Liber descriptaribus eedesiastias de Johannes Trithemius, publicada en 1492.12

Antes de ésta existieron intentos por crear bibliografías, como es el caso delas listas de libros de las bibliotecas catedralicias, que se hacían con el fin de organizar sus colecciones. Sin embargo éstas fueron simples listas que se hicieron por razones de inventario pero que no facilitaban el acceso a su contenido, pues contenían una descripción breve elaborada sin uniformidad y sólo se mencionaba autory título más algunas características físicas del libro, como tipo de letra en el queestaba escrito, si tenía ilustraciones, etcétera. ${ }^{13}$

En opinión de Murison ${ }^{14}$ es a partir de la aparición de las bibliotecas públicas cuando los documentos son organizados de manera sistemática, aspecto que retomaremos más adelante.

10 Ciudad que se ubicaba cerca de las costas mediterráneas de Asia Menor.

11 Escolar, Sobrino, Hipólito. Histaiadelasbiblideeas Fundación G ermán Sánchez Ruipérez, 1990. p. 149.

12 Theodore Bestermann. Lesdautsdelabibliogaphiemóthodique3ª ed. Paris: La Palme, 1950. p. 19-23.

13 Lerner., Fred. Thestary oflibranies: fromtheinuention of witingtothecomputerage New Y ork: Continum, 1998. p. 94.

14 Murison, W.J.Thepubliclibrary. itsorigins, purpose, andsigificance 3를. London : Clive Bingley, 1988. p. 27. 
Posteriormente haría su aparición labibliotecanacional, que si bien es vista como figura ideal para asegurar la conservación y difusión de la cultura nacional, 15 no puede verse como un tipo de biblioteca basada en el modelo de acumulación. Sobre todo porque tiene una cualidad que la distingue: su función es generar la bibliografía nacional, el registro de las obras que posee.

En labiblioteca nacional subyace la idea de universalidad perseguida con anterioridad en aquellas bibliotecas cuyo fin principal era la acumulación de la producción bibliográfica universal. Pero esta idea también fue continuación de los intentos hechos por algunos individuos ${ }^{16}$ por registrar esta producción, tanto a través de repertorios bibliográficos como de catálogos de bibliotecas. A partir de entonces, han surgido diversos proyectos de organismos internacionales dirigidos a la creación de repertorios nacionales y universales que permitan un control bibliográfico.

\section{La biblioteca bajo el modelo de libre acceso}

Como bien se sabe, hasta antes del siglo XV los manuscritos se reservaban a una élite circunscrita de individuos letrados, ${ }^{17}$ lo que se reflejaba en la conformación de las bibliotecas, pues las de la Edad Media estaban pensadas para resguardar el saber para unos cuantos eruditos, que en su mayoría eran monjes pertenecientes a las diferentes órdenes que tenían a su cuidado los acervos.

Posteriormente, además del papel asignado a la biblioteca como resguardadora de libros, comenzó a dársele importancia a su acceso, cuestión que tomaría mayor relevancia con el paso del tiempo.

Elmodelo de acceso se refiere ala apertura dela biblioteca, en el sentido de permitir su uso a todo aquel que asíl lo requiriera. Algo fundamental en este modelo es la aparición de la biblioteca pública como medio para el libre y amplio acceso a los documentos.

Pese a que es impreciso el momento en el que aparece el concepto de biblioteca pública, Lerner considera que éste se origina en la época de la Reforma Protestante, contexto dentro del cual se creyó necesario que cada miembro de la sociedad recibiera una educación que le permitiera leer e interpretar la Biblia lo cual exigía un acceso más amplio a la enseñanza y a los libros. ${ }^{18}$ La interpretación individual de las Escrituras, sin mediación de la autoridad romana, les confirió al libro y a la lectura la categoría de instrumentos de fe.

Aun cuando con la invención de laimprenta un mayor número de personas pudo tener acceso a los libros, muchos estaban escritos en latín, lo que se produjo fue un incremento de literatura y la traducción a otras lenguas. De hecho en las regiones

15 Y reflejó una política de adquisición enciclopédica que permaneció hasta el fin del siglo XIX.

16 Como Gabriel Naudé o Paul Otlet.

17 Eco, Umberto. El pareirirdeloslibros En: 20 CongresodelaUniónIntemadional deEditares Barcelona: UIE, 1996. p. 4.

18 Lerner. Op Cit, p. 138. 
donde dominaron los impulsores de la Reforma Protestante se estableció el derecho de ofrecer a la población ejemplares de laBidiay otro tipo de textos religiosos escritos en lenguas vernáculas. Así el número de libros en estas lenguas nacionales se incrementó y más gente pudo tener acceso a ellos.

Por eso se le suele atribuir al protestantismo un talanteliberal y abierto en materia de lecturas y una activa política de promoción del libro y de las bibliotecas; la dotrina de libreexamenha parecido un sólido fundamento para este juicio.

Para otros expertos, la verdadera biblioteca pública aparece en E uropa hasta fines del siglo XIX, pero bajo la influencia de las ideas gestadas durante la Revolución Francesa, ya que con los principios de so beranía popular y de propiedad nacional de los bienes culturales proclamados en laDedaracóndelosDeechosda Honbre(1798), se impulsó una importante labor en las bibliotecas públicas.

Ejemplo de ello fue cuando el obispo constitucional de Blois, Henri Grégoire, uno de los más destacados ideólogos revolucionarios en el campo de las bibliotecas, presentó a la Convención Nacional su Rapport surlabibliographie, en el que afirma la justa necesidad de repartir los bienes culturales, incautados a la Iglesia refractaria, a la nobleza y a los emigrados, en provecho de todos los ciudadanos. Los D epósitos Nacionales, creados para recoger y distribuir esos objetos científicos en régimen de igualdad, ${ }^{19}$ acarrearon serias dificultades al provocar la adecuación de los fondos para las bibliotecas populares y suscitar las tropelías que con ellos cometieron personas desconocedoras del valor de esos materiales.

Un texto menos conocido, la Mémiresurl'usageqúon peat fairedes livresnationaux (1772), redactado por el sacerdote constitucional Francois-X avier Laire, repetía las ideas de la revolución sobre el libro y la cultura nacionales. O tro texto que publicó fue un Projet defomation des biblidhequesnationales(populares públicas), donde pedía que se entregaran al público las colecciones de libros pertenecientes o vinculadas a los obispos. Un decreto de la Convención de 1795, ordenaba:

Poned una bibliotecajunto a la sociedad popular principal de cada distri-

to. La literatura y las ciencias deben aliarse con las virtudes cívicas. ${ }^{20}$

Unaacción perdurable de los revolucionarios fue la nacionalización de las bibliotecas reales, acción que fue ejemplo para otros países. D e cualquier manera es destacable su deseo por ampliar el acceso a la educación y la cultura, valores en los que está involucrado el acceso a los documentos escritos.

En Inglaterra la lectura pública, en su etapa inicial, nació en las escuelas. Promovida por el clero protestante para la instrucción de sus miembros, pasó luego a las trece colonias (actualmente Estados Unidos).

La nueva educación y el propósito de altruismo del momento dirigieron a la biblioteca a través de dos caminos: uno cultural, encaminado al apoyo de la educación y 
otro, que originado en la iglesia, favoreció la promoción y difusión del conocimiento. La convergencia de esos dos momentos marcó la génesis del modemo concepto de biblioteca pública. ${ }^{21}$

Este movimiento iniciado en Europa (sobre todo en la Gran Bretaña) también adquirió fuerza en Estados Unidos durante el siglo X IX, aunque bajo propósitos un tanto diferentes. Sin embargo, puede observarse un rasgo común en los inicios de la biblioteca pública: el principio del libre acceso al contenido de los libros para todos. Este principio se conoce hasta nuestros días como el pinipiodeaccesoabietoy uno de los autores más representativos en su establecimiento es Ranganathan.22

Labiblioteca pública se convertiría así en un instrumento para la educación y, en el caso de Inglaterra, en un medio para elevar el nivel educativo y en consecuencia el nivel de vida de las clases trabajadoras, así como para facilitar la recreación para las clases medias.

Hasta nuestros días las misiones de la biblioteca pública guardan gran relevancia, so bre to do en Estados Unidos e Inglaterra, pero han adquirido otros matices a partir del creciente uso de las tecnologías de la información

\section{La biblioteca en el modelo de cooperación bibliotecaria}

D urante las décadas de 1960 y 1970 se incrementó tanto la producción editorial, que las bibliotecas académicas tuvieron dificultades para adquirir todo el cúmulo de publicaciones. ${ }^{23} \mathrm{O}$ tro problema fue el relativo a los reducidos espacios que tenían dichas bibliotecas.

Una solución propuestafue hacer una selección minuciosa de los materiales: sólo aquello que fuera verdaderamente útil para su comunidad sería adquirido. Esto podría dejar de lado muchas publicaciones y libros que sin ser de uso frecuente también podrían requerirse en alguna ocasión. La solución que se eligió fue que las bibliotecas académicas compartieran sus colecciones. ${ }^{24}$

\section{LOS SERVICIOSEN LA BIBLIOTECA TRADICIONAL}

Los modelos anteriores nos permiten identificar los servicios bibliotecarios y de información a los que ha dado origen la biblioteca tradicional.

\section{Los servicios en el modelo de acumulación}

Poco hay que decir sobre los servicios de labiblioteca orientadaa la acumulación, pues presenta un uso casi nulo de los documentos. Su función básica es la preservación de la memoria escrita, pero no ofrece servicios a través de los cuales pueda tenerse acceso a esa memoria.

21 Thompson, James. A histay of thepiniples of libraiandip Londres : Clive Bingley, 1974. p. 70.

22 Ranganathan, S. R. Fivelausoflibrarysaiere Madras: London, Madras Library Association, 1931.

23 Brophy, Peter., Op Cit., p. 44.

24 Ibidam 
Aunque este modelo es la imagen más fiel de lo que podría ser una biblioteca universal, lo es en razón de la cantidad de documentos que posee físicamente, pero no en razón del acceso a la información.

Por ejemplo, durantela época de las bibliotecas monacales la ignorancia generalizada de la población y el hecho de que la I glesia se mostrara tan celosa de su misión como guardiana de la doctrina cristiana, provocaron que la biblioteca se limitara a resguardar libros.

\section{Servicios del model o basado en el registro de la información}

Puede aseverarse que los servicios bibliotecarios surgen en el momento que comienza a registrarse la información. Anteriormente durante el apogeo de las bibliotecas monacales eran los eruditos quienes servían de medio para identificar las obras útiles para el usuario, pero en la época en que estos hombres tenían a su cargo las bibliotecas, existía una gran limitación en al acceso a las obras.

En la época de las bibliotecas catedralicias los préstamos también eran muy restringidos y se concedían mediante fianzas. De cualquier manera es indiscutible su aportación para iniciar ese tímido acercamiento a la población y en consecuencia su contribución a un acceso más amplio a los documentos escritos.

No es sino hasta la aparición de las universidades cuando se crean catálogos en las bibliotecas. ${ }^{25}$ Se concibe también entonces la sección quepodríallamarse de consulta - con libros encadenados- y otra formada por los libri distribuendi, que se prestaban a los profesores.

Sin embargo este tipo de biblioteca representó tan solo un modesto modelo inicial: el logro de un acceso amplio a los acervos de las bibliotecas. Puede decirse que con esta biblioteca aparecen incipientes servicios de préstamo y de consulta.

En la biblioteca de la Sorbona, por ejemplo, los libros se usaban dentro del edificio de la biblioteca. Y en caso de utilizarlos fuera debían devolverse al final de ese mismo día y habría que dejar un depósito equivalente al valor del libro.

O tro aspecto que dio pie al desarrollo de los servicios bibliotecarios fue el nacimiento de las sociedadescientíicaso academias, que crearon sus propias revistas, como la A cademia dei Lincei,(1603) en Roma, la Royal Society (1663) en Londres y la Academie Royale des Sciences (1666) en París.

En esa etapa los bibliotecarios profesionales favorecieron la conversión de las bibliotecas en instrumentos de trabajo al servicio de la cultura superior. Se crean también entonces los catálogos alfabéticos de autores y de materia, distintos para impresos y manuscritos. Al catálogo de materia se le daba mayor importancia porque los lectores lo utilizaban con mayor frecuencia. El de autores tenía una consideración secundaria: conocer las obras de un autor que hay en la biblioteca para 
evitar la compra de obras repetidas y facilitarle a los interesados la relación de o bras en él dispuestas. ${ }^{26}$

Junto con toda esa nueva concepción de la biblioteca surgieron notables estudiosos que sentaron las bases de la biblioteconomía moderna. Entre ellos destaca Charles Coffin Jewett y Charles A mmi Cutter, esteúltimo, creador de un sistema de clasificación que aunque no fue totalmente desarrollado se utilizó parcialmente en la organización de la famosa Biblioteca del Congreso. También se distingue Melvil D ewey, a quien se debe el sistema de Clasificación D ecimal que lleva su nombre y por quien se establecen los primeros cursos de enseñanza profesional. Todos ellos tuvieron una participación activa en la creación de la AmeicanLibraryAssociation así como de la revista LibraryJaumal, gracias a las cuales se unificó y difundió el pensamiento moderno de la ciencia bibliotecaria.

\section{L os servicios en el modelo de libre acceso}

A sociada con la aparición de la ciencia bibliotecaria estála concepción de los servicios públicos de información, que marcarán muchas de las características de los servicios que hoy conocemos.

En el contexto actual la biblioteca pública tiene la misión de asegurar la igualdad de acceso al conocimiento con base en un espíritu de justiciay libertad. ${ }^{27} \mathrm{D}$ eacuerdo con el ManifiestodelaUNESCO, la biblioteca pública es un centro local de información que le facilita a sus usuarios todas las clases de conocimiento e información. ${ }^{28}$ Entrelas misiones que establece la UNESCO , se contempla quela biblioteca pública:

* Sea gratuita y

* Financiada por los gobiernos nacional y local

Y por tanto que la facilitación de los servicios tiene relación directa con la gratuidad de los servicios.

D e aquí surge el concepto de servido pública que en gran medida prevalece en nuestros días. La gratuidad del servicio es una característica esencial, ya que por el uso de las colecciones de bibliotecas tradicionales no se exige cobro; los costos derivan más bien de servicios adicionales como el de reprografía.

Fosket asevera que el servicio bibliotecario ha respondido a una serie de condiciones particulares que no dependen del tipo de biblioteca de la que se trate; sin embargo la idea de acceso amplio e irrestricto, alcanzó su punto máximo con la biblioteca pública, la cual condicionó la idea de seviaiopública 29

26 Escolar. Op Cit, p. 311.

27 Ramos Simón, Luis Fernándo. Tanificaiónygatuidadend ámbitodbamental. En: Doumentacióndelas Cienias dela Infomaión n. 19. (1996). p. 245.

28 Missions of thePublicLibrary. Disponible en: http:/ / www.unesco.org/ webworld/ libmanif/ plmissi.html

29 Foskett., Op Cit, p. 12. 


\section{Investigaaón Biblictedóǵgia v. 15 N o. 31 julio/ diciembre de 2001}

\section{L os servicios basados en la cooperación bibliotecaria}

Este modelo se hace presente con gran fuerza en la década de los setenta, cuando se populariza el uso de bases de datos en línea, sobre todo en las bibliotecas académicas y especializadas.

En este periodo se presentó una gran producción editorial que no podía ser adquirida en su totalidad por las bibliotecas, tanto por falta de presupuesto como de espacio para albergar esos materiales. La cooperación bibliotecaria surgió así como una alternativa.

Pero para que esta cooperación fuera efectiva se hacía necesario contar con medios que permitieran identificar qué materiales tenían las demás bibliotecas. Es aquí en donde el catálogodeuniónjuega un papel importante;30 con el uso de tecnologías este catálogo representó significativos ahorros en la adquisición y búsqueda de materiales.

En tal contexto también se dio la generación de diversos productos y servicios, muchos de ellos basados en el uso de las bases de datos. Entre estos servicios destacan la diseminación selectiva de la información ${ }^{31}$ y el servicio de entrega de documentos.

El servicio depréstamo interbibliotecario se reconocería también como un aspecto fundamental dentro del modelo de cooperación bibliotecaria, ${ }^{32}$ pues si bien dicho servicio ya estaba presente en la biblioteca pública, su demanda no era tan grande.

A la par que las bibliotecas comenzaron a ofrecer sus catálogos en línea, algunos productores vendieron sus bases de datos a las bibliotecas, como el caso de OCLC (O nline Computer Library Center). Este sector comercial ofrecería entonces servicios de indización y resumen, primero en las ciencias aplicadas y después en las ciencias sociales y las humanidades. 33

El uso de discos compactos se convertiría posteriormente en una alternativa para la búsqueda y recuperación de información.

\section{CONCLUSIONES}

A partir de lo expuesto, pueden hacerse las siguientes consideraciones:

Los servicios bibliotecarios surgen gracias al modelo de biblioteca tradicional que se basa en el registro, pues en el modelo anterior de acumulación no se establecía ese puente que permitiera el acceso a los materiales de la biblioteca. Sólo una pequeña élite podía entonces hacer uso de los materiales y era el erudito quien podía guiar al usuario en su búsqueda.

Así, los servicios bibliotecarios surgen como tales a partir de la sistematización de los materiales que conforman una colección. En este sentido los repertorios bibliográficos jugaron un papel importante, al ser los primeros medios a través de los

30 Ilid, p. 45.

31 Lancaster F., Wilfrid. Infomationrenieal today. Virginia: Information Resources Press, 1993. p. 21.

32 Martin, Susan K. Libraryntworks, 1986-87: libraniesinpartneship - New Y ork : K nowledge Industry Publications, 1986. p. 12.

33 Ilid, p. 29. 
cuales se podían conocer los materiales que conformaban una colección. Pero el catálogo ocupa un lugar central en el establecimiento de los servicios, en tanto que sistematiza los materiales.

A partir delas bases que sentaron los estudiosos para la organización de los materiales, se sistematizan también los servicios; es decir, pueden darse tipos de servicios más definidos, como los de préstamo y consulta.

Con el modelo de acceso surge la idea del servicio público en dos sentidos: la apertura de la biblioteca para to do aquel que requiera utilizarla y la gratuidad del servicio. Este modelo aún persiste en muchas de las bibliotecas actuales.

El modelo de cooperación bibliotecaria dio origen a los servicios deinformación, ya que los materiales identificados a través de los catálogos de unión pueden, 0 no, ser parte de la colección de la biblioteca desde la cual se consulta el catálogo.

La cooperación de la que se habla se da en el sentido de compartir recursos documentales. Si bien es cierto que esta cooperación se hacía presente con anterioridad a través del préstamo interbibliotecario, es con estemodelo cuando se convierte en un servicio sustancial.

Para alcanzar el florecimiento de la cooperación que se generó en la década de los stenta, fue de gran utilidad la aparición de las bases de datos y de los catálogos en línea. D esde entonces esta biblioteca se conocerá como biblioteca automatizada.

Comienza entonces la biblioteca automatizada a darle prioridad a la identificación de los documentos más que a su posesión física.

Además aparecen servicios como el de diseminación selectiva de la información y el de entrega de documentos en línea (y posteriormente vía fax).

En general puede afirmarse que en la biblioteca tradicional que hoy conocemos, se combinan uno o más de los modelos mencionados y que ello da lugar a imágenes diversas de esta biblioteca.

En labiblioteca tradicional de nuestros días, existen tanto servicios bibliotecarios como de información. Ambos se complementan y conforman una unidad de servicios y no pueden ser disociados.

Finalmente cabe mencionar que a partir de lo aquí estudiado se está en mejor condición para analizar si los servicios que se observan en el medio digital son inéditos o si poseen características diferentes cuando se compara con los servicios de la biblioteca tradicional. Claro está, se requiere de un estudio previo que muestre los servicios que puede ofrecer la biblioteca digital.

\section{O BRAS CONSULTADAS}

Arashanipalai Neelameghan. Cocpeaciónyasisteniaintemadionales En:Informemundal sobrelainformacón 1997-1998/ Y vesCourrier y Andrew Large, ed. - Madrid : UNESCO : CIND OC, 1997.

Brophy, Peter. Thelibraryin thetwenty first century: newsenvices for theinformationage - London : Library Association Publishing, 2001. 
Buckland, Michael. ReeksigningLibraryServices A ManifestoD isponibleen: http/ / sunsitebokkdey.edv/ Literaturel Library/ Reeksigning htm.html

Eco, Umberto. El parvenirdeloslibros. En: $20^{\circ}$ CongesodelaUniónIntemado nal deEditares Barcelona : UIE, 1996.

Escolar, Sobrino, Hipólito. Histariadelasbiblidteeas - Fundación G ermán Sánchez Ruipérez, 1990.

Foskett, D J. Infomationserviceinlibranies - India : A kashdeep, 1992.

Lancaster F., Wilfrid. Informationrenieval today. - Virginia : Information Resources Press, 1993.

Lerner., Fred. Thestaryoflibranies: fromtheinvertionofwitingtothecomputerage - New York : Continum, 1998.

Martin, Susan K. Libraryndworks, 1986-87: libraniesin partneship - New Y ork : Knowledge Industry Publications, 1986.

Missions of thePublicLibrary. D isponible en: http/ / unurunescaarg webword/ libmanif/ plmissi.htm

Mitchell W., Bede. Access: thekeytopublicserice - p. 1-22. - En A cessservcesinlibranes: newsdutionsfor cdletionmanagemet/ G regg Sall, editor. N.Y : Hartworth, 1992.

Murison, W. J. Thepudiclibrary: itscrigns, purpose andsigificance - $3^{\mathrm{a}} \mathrm{ed}$. London : Clive Bingley, 1988.

Ramos Simón, Luis Fernándo. Tanificaiónygatuidadend ánbitodboumental. En: Dœumentacoón delas Cienaias dela Información - n. 19. - (1996).

Ranganathan, S. R. Fivelausfflibrarysaiene - Madras : London, Madras Library Association, 1931.

Theodore Bestermann. Les dauts dela bibliogaphiemóthodique . - 3a ed. Paris : La Palme, 1950.

Thompson, James. A histaydfthepiniples flibranianship - Londres : Clive Bingley, 1974.

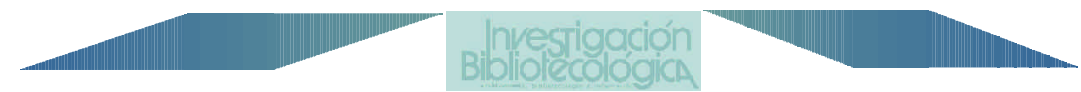

